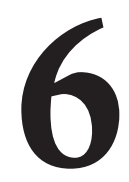

\title{
PARTICIPACIÓN EN LAS REDES SOCIALES DEL ALUMNADO DE EDUCACIÓN SECUNDARIA
}

\section{(PARTICIPATION IN SOCIAL NETWORKS BY SECONDARY SCHOOL STUDENTS)}

Francisco Javier Ballesta Pagán

Josefina Lozano Martínez

Mari Carmen Cerezo Máiquez

Irina Sherezade Castillo Reche

Universidad de Murcia

DOI: $10.5944 / e d u c X X 1.26844$

\section{Cómo referenciar este artículo/How to reference this article:}

Ballesta Pagán, F.J.; Lozano Martínez, J.; Cerezo Máiquez, M.C. y Castillo Reche, I.S. (2021). Participación en las redes sociales del alumnado de Educación Secundaria. Educación XX1, 24(1), 141-162, http://doi.org/10.5944/educXX1.26844

Ballesta Pagán, F.J.; Lozano Martínez, J.; Cerezo Máiquez, M.C. \& Castillo Reche, I.S. (2021). Participation in social networks by secondary school students. Educación XX1, 24(1), 141-162, http://doi.org/10.5944/educXX1.26844

\section{RESUMEN}

En este trabajo se presentan los resultados de una investigación realizada con la finalidad de valorar la participación en las redes sociales del alumnado de $3^{\circ}$ y $4^{\circ}$ de Educación Secundaria (ESO). El objetivo ha sido conocer el acceso y uso que efectúan en su vida cotidiana, y las diferencias que se aprecian en función del género, origen (autóctono-extranjero) y necesidad específica de apoyo educativo (NEAE). El diseño metodológico ha sido mixto aplicando un cuestionario tipo Likert, con cinco opciones de respuesta, a 2734 alumnos de 15 centros en la Comunidad Autónoma de la Región de Murcia y, complementada con la aplicación de la técnica "Phillips 66" a 157 alumnos seleccionados de cinco de los centros, en función del mayor o menor uso de estas tecnologías. Los resultados indican que el 
acceso a las redes sociales es mayoritario, aunque menor en el alumnado extranjero y con necesidad específica de apoyo educativo. La participación no está orientada únicamente a intereses personales, sino a necesidades sociales afectivas y relacionales, destacando el entretenimiento, la presencia de amigos y la necesidad de conocer gente nueva. No existe una percepción negativa sobre su consumo que influya, desfavorablemente, en las tareas de estudio, ocio y relación personal. Se aprecian diferencias significativas entre el alumnado relacionadas con la percepción que tienen sobre la mejora de las relaciones con los amigos, debido al uso de las redes sociales. Así, se identifica un sentimiento menos positivo del alumnado con NEAE, mientras que el alumnado extranjero las utiliza, principalmente, para conocer gente nueva. En cuanto al género, son ellas las que llevan más tiempo participando con la finalidad primordial de contactar con quienes no ven, manifestando tener discusiones con amigos y familia por su uso, y reconociendo tener más preocupación ante la posibilidad de no utilizarlas.

\section{PALABRAS CLAVE}

Redes sociales, educación secundaria, inmigración, género, necesidades educativas especiales

\section{ABSTRACT}

This study explores the participation in social networks of Year 3 and Year 4 Compulsory Secondary School students with regard to frequency of access and use, gender, ethnicity and special educational needs. The study adopted a mixed-method research design, surveying 2734 students from 15 Secondary Schools in the Region of Murcia (Spain) who were asked to complete a questionnaire on a 5-point Likert scale. Data collection was complemented with the application of the "Phillips 66" technique to 157 focus students selected depending on their frequency of use of these technologies. The results reveal a widespread use of social networks, though foreign and special needs students use them to a lesser extent. Student engagement with social networks is not solely oriented to meeting personal interests, but socioaffective and relational needs such as entertainment, being in touch with friends and meeting new people. These students do not hold the perception that social networks might negatively impact their academic performance, leisure time and personal relationships. Statistically significant differences were reported for students' perceptions about the usefulness of social networks when it comes to improving existing friendships. In this respect, students with special needs feel less positive, while foreign students use social networks mainly for meeting new people. As for gender, women spend more 
time on social networks with a view to keeping in touch with people they do not normally see. They also report having problems with relatives and friends regarding their use of social networks, and they feel more anxious at the thought of not having access to them.

\section{KEY WORDS}

Social networks, secondary education, immigration, gender, special education needs

\section{INTRODUCCIÓN Y ESTADO DE LA CUESTIÓN}

La irrupción de las tecnologías en nuestra vida ha cambiado la manera de relacionarnos, favorecida por el avance en la utilización de dispositivos móviles conectados a Internet y, de forma especial, al móvil que se ha convertido en el medio preferente que, de forma vertiginosa, ha incrementado su consumo entre la población y, en especial, los más jóvenes (AIMC, 2019; Fundación Telefónica, 2019; INE, 2019). Esta revolución digital ha propiciado una mayor interacción social, aunque al mismo tiempo, puede producir dependencia y riesgos en los más jóvenes (Soler et al., 2018). La generalización en el uso del móvil para acceder a las redes sociales y a la navegación está manifestando nuevos hábitos y rutinas comunicativas entre los jóvenes consumidores que lo tienen como un aliado en su vida, como facilitador para el desarrollo de relaciones sociales, encontrar nuevos amigos, o reforzar vínculos existentes. En el caso de los jóvenes entre 14 y 19 años este dispositivo se ha convertido en una herramienta imprescindible para la socialización y lo usan de forma habitual, pasando la mayoría de su tiempo frente a él, ocupando las redes sociales la mayor parte de su actividad, junto a la mensajería, música, vídeos, descargas desde las múltiples apps (Gewerc et al., 2017).

De igual modo, la mayoría de adolescentes, a partir de los 10 años, se siente más competente digitalmente que sus padres y madres (Garmendia, et al., 2016) y es a partir de la etapa de Educación Secundaria, cuando se comprueba que los roles educativos parecen haberse invertido ya que, para el alumnado, estos instrumentos son parte de su medio natural, mientras que los adultos han requerido de un esfuerzo extra para llegar a ser competentes (Sola et al., 2019). Esta práctica digital ha cambiado la forma de comunicación grupal e interpersonal entre adolescentes y está modificando el modo en que se relacionan, poniendo de manifiesto que la finalidad comunicativa de la participación en las redes sociales, utilizadas mayoritariamente entre los más jóvenes, es una realidad que les permite 
incorporarse a una macro comunidad preexistente en la que casi cualquier relación entre sus miembros es posible, y donde la necesidad de compartir una identidad pública viene definida por la funcionalidad de este entorno virtual que le brinda la posibilidad de explorar y construirla entre sus coetáneos y su grupo de iguales (Boyd \& Ellison, 2008; Pacheco et al., 2018).

Los resultados de investigaciones previas muestran que los estudiantes de Secundaria buscan una mayor presencia social y velan por su propia imagen (García-Ruiz et al., 2018). Esta es la razón que justifica una participación continuada de los jóvenes en las redes sociales, debida fundamentalmente a la creciente importancia dada a la construcción de su propia identidad digital que los lleva a buscar su satisfacción personal y bienestar social, por lo que procuran expresarse y relacionarse, casi exclusivamente, a través de este medio (González y López 2018, Yau, \& Reich, 2018). Los jóvenes participan en las redes sociales, fundamentalmente, por las relaciones que ofrecen más que por la transmisión de información, por lo que su valor va unido a la posibilidad que les brindan de tener nuevos amigos que es más que tener una lista de contactos, favoreciendo la creación de vínculos sociales existentes, de amistad o generar otros nuevos (Manca \& Ranieri, 2017). La mayoría de los usuarios justifica su pertenencia a una red social en el hecho de que le permite mantener contacto con sus amigos y/o conocidos, con los que ve con frecuencia, en especial los compañeros de clase y, con bastante distancia, para hacer nuevos amigos por lo que la motivación para participar en ellas y tener varias cuentas lleva implícita la necesidad de potenciar y mantener las relaciones de amistad fuera de la red social (Rubio et al., 2019; Vizcaíno et al., 2019).

Esta participación en las redes sociales gira en torno a la necesidad de comunicarse y lo hacen desde la iconicidad, sus conversaciones giran en torno a la imagen, en todos sus formatos, proyectando sus sentimientos, emociones y dedicándole una gran inversión de tiempo, siendo conscientes, y en ocasiones ingenuos, de que tienen la oportunidad de mostrar un perfil de si mismos, ante su grupo de iguales, identificándose a través del intercambio de mensajes entre amigos y conocidos, permitiéndoles obtener información de interés y compartirla entre los seguidores y los coetáneos que conforman sus referentes (Ibrahim et al., 2017). El valor que tienen las redes sociales para la socialización de los adolescentes y el inicio temprano es una característica común que se viene manteniendo desde ya hace algunos años, iniciando a los 12 años su actividad, con el primer perfil y haciéndolo de forma continuada, confirmándose que en torno a esa temprana edad, conforman un grupo importante que inicia la participación (Bernal y Angulo, 2013; Colás et al., 2013; Garitano et al., 2019; Greenhown \& Askari, 2017; García et al., 2013). 
El presente artículo pretende dar respuesta a estas cuestiones. Para ello se plantea como objetivo general conocer el acceso y uso en las redes sociales del alumnado de $3^{\circ}$ y $4^{\circ}$ de Educación Secundaria Obligatoria (ESO) de la Región de Murcia en función del género, origen (autóctono- extranjero) y necesidades especiales (con o sin necesidad específica de apoyo educativo), para comprender el consumo que realiza en su vida cotidiana.

\section{MÉTODO}

\section{Objetivos específicos}

Para lograr el objetivo general expuesto anteriormente, nos plantemos los siguientes objetivos específicos:

1. Valorar la participación en el acceso y uso de los jóvenes atendiendo a la pertenencia a los grupos establecidos.

2. Analizar las ventajas e inconvenientes de la participación en las redes sociales.

\section{Muestra}

La muestra productora de datos de la investigación ha sido el alumnado de $3^{\circ}$ y $4^{\circ}$ de la ESO escolarizados en 15 centros de la Región de Murcia. La selección muestral ha sido de tipo estratificada al azar bietápica, en la que el único criterio de selección ha sido la localidad, pretendiendo que nuestro universo quedara representado proporcionalmente a su distribución en toda la región. Esta muestra de 2734 encuestados, se corresponde con un nivel de confianza del $97 \%$, ofreciendo un \pm 2.89 de error muestral.

\section{Técnicas, instrumentos y procedimiento}

En relación con las técnicas e instrumentos se ha utilizado el método descriptivo encuesta, a través de un cuestionario como instrumento de recogida de información. Este se estructuró en seis dimensiones: Identificación, Internet, Redes Sociales, Teléfono Móvil, Videoconsola y Televisión, formulándose un total de 74 ítems. En este artículo abordamos la tercera dimensión referida a las redes sociales, para valorar el acceso y preferencias del alumnado sobre este nuevo canal comunicativo. Para ello agrupamos 17 ítems referidos a: participación, finalidad del uso y tiempo de utilización, actividad realizada y percepción sobre la influencia que tiene en la relación con la familia, los amigos y la realización de tareas escolares. 
Una vez elaborado el cuestionario, lo sometimos primeramente a un juicio de expertos para su validación y evaluación, y posteriormente a la evaluación del contenido del cuestionario que se realizó en dos fases: la primera centrada en valorar el constructo u objeto de estudio, y la segunda en la valoración del contenido de cada ítem. La fiabilidad (consistencia interna) del instrumento es de .784 y, en concreto, la de la dimensión de estudio muestra un Alpha de Cronbach de .746. Tras la recogida de la información se procedió al análisis cuantitativo de los 2734 alumnos, en el cual se realizó un análisis descriptivo con las correspondientes tablas de frecuencias y porcentajes y un análisis bivariable utilizando las pruebas de significación, con el cálculo del estadístico Chi cuadrado de Pearson.

Con la finalidad de complementar los datos obtenidos en el cuestionario, aplicados a los 2734 alumnos y alumnas de los 15 centros ubicados en distintas zonas, se seleccionó a 157 alumnos (un 6\% de los alumnos encuestados) de 5 centros educativos de la región para participar en la técnica "Phillips 66". Estos centros se seleccionaron atendiendo al criterio de estar por encima o por debajo de la media en el uso de las TIC, evitando en esa selección la coincidencia de centros en la misma localidad. Tras esa selección se realizó la dinámica de grupo mediante dicha técnica para profundizar en cuestiones relacionadas con las motivaciones, efectos, problemas y creencias en torno al uso y consumo que hacen de las nuevas tecnologías.

El procedimiento seguido para llevar a cabo la técnica "Phillips 66" en las aulas de $3^{\circ}$ y $4^{\circ}$ de Educación Secundaria, consistió en formar grupos de 6 alumnos, los cuales disponían de 6 minutos para debatir, en pequeño grupo, con relación a cada una de las preguntas planteadas sobre el uso y participación en las redes sociales. Cada pequeño grupo tenía un portavoz, que recogía las aportaciones de los compañeros, y que las exponía al grupoclase en una segunda fase. Estas valoraciones se recogieron por parte del investigador para ser estudiadas posteriormente. Para el análisis de esta técnica se utilizaron una serie de categorías, establecidas a priori, que tenían como finalidad complementar la información recabada en las distintas dimensiones del cuestionario.

\section{RESULTADOS}

Con respecto a los datos de identificación de la muestra productora de datos de la investigación, y con relación, concretamente, al curso escolar, hay que afirmar que está repartida por igual, dado que un 53\% es alumnado de $3^{\circ}$ y el $47 \%$ cursan $4^{\circ}$ de la ESO. En lo relativo a la distribución por género, estamos ante porcentajes muy próximos, siendo un $1.4 \%$ mayor la presencia de chicos en la investigación. Si atendemos al lugar de 
nacimiento de los jóvenes, la información recogida, a fin de hacerla más manejable la hemos reducido en dos categorías: estudiantes que han nacido en el país y estudiantes que han nacido fuera de nuestras fronteras, así podemos identificar, en base a esta clasificación, cómo un $80.7 \%$ del total son autóctonos y un $19.3 \%$ son extranjeros. En cuanto al alumnado con necesidad específica de apoyo educativo (NEAE), el $82.2 \%$ de los alumnos no presentan necesidades, siendo un $17.8 \%$ los que sí presentan.

\section{Acceso a las redes sociales}

Centrándonos en la participación en las redes sociales cabe resaltar, tal y como podemos observar en la figura 1, que casi el 90\% de los adolescentes de $3^{\circ}$ y $4^{\circ}$ de ESO en la Región de Murcia acceden a ellas; de estos, el 71.4\% lo hace con un perfil propio, el $4.1 \%$ sin un perfil propio y el $14.5 \%$ utiliza, tanto uno como otro. Solo un $10 \%$ de la muestra no participa aún en ellas. $\mathrm{Si}$ atendemos a las diferencias entre grupos, tal y como podemos observar en la figura 1, existe una diferencia significativa en el acceso a redes sociales en cuanto al origen del alumnado $\left(\chi^{2}(3)=59.458 ; \mathrm{p}=.000\right)$ en el que son más los autóctonos que acceden con perfil propio y participan más que los extranjeros. Lo mismo ocurre con el alumnado que presenta necesidades específicas de apoyo educativo (NEAE) $\left(\chi^{2}(3)=28.433 ; \mathrm{p}=.000\right)$; la mayor diferencia la observamos en aquellos que reconocen no participar en las redes sociales, siendo casi el doble de porcentaje (16.2\%) de alumnos con NEAE quienes defienden esta opción, frente al 8.6\% de los alumnos sin estas necesidades específicas.

Figura 1

Acceso a las redes sociales

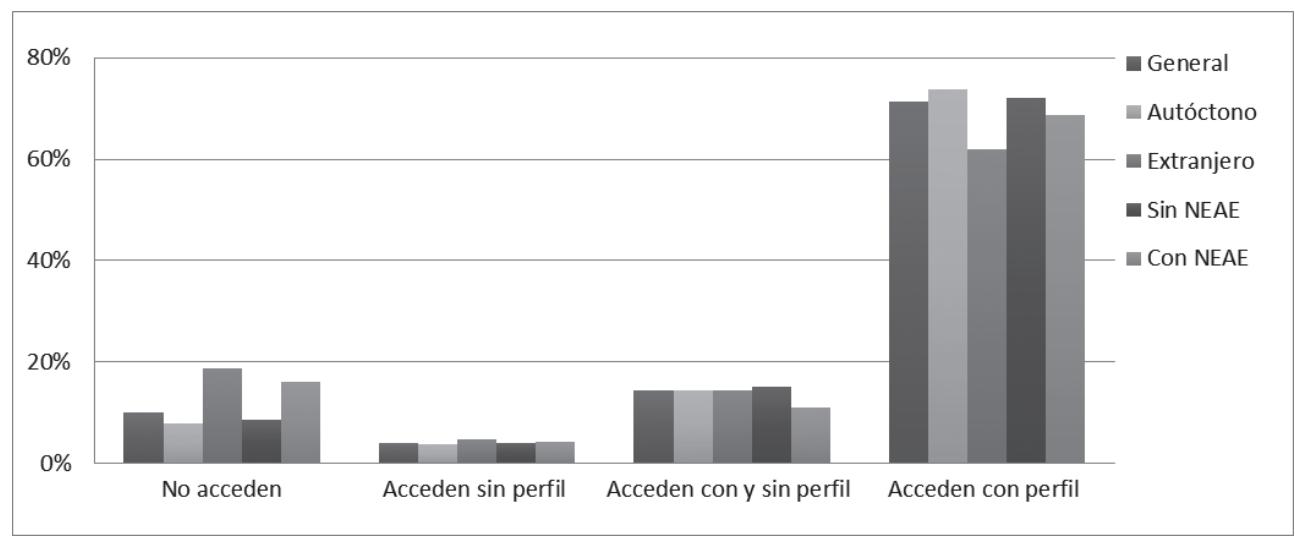




\section{Tiempo de participación}

La gran mayoría del alumnado participa desde hace tiempo en las redes sociales, tan solo el $11.7 \%$ lleva menos de un año, como se puede ver en la figura 2. En cuanto a las diferencias por grupos, las chicas llevan más tiempo participando $\left(\chi^{2}(3)=15.646 ; \mathrm{p}=.001\right)$ que los chicos. Asimismo, del alumnado que lleva haciéndolo más de tres años, son los alumnos extranjeros $(25.4 \%)$ por encima de los autóctonos $\left(\chi^{2}(2)=57.645 ; \mathrm{p}=.000\right)$.

Figura 2

Tiempo participando en las redes sociales

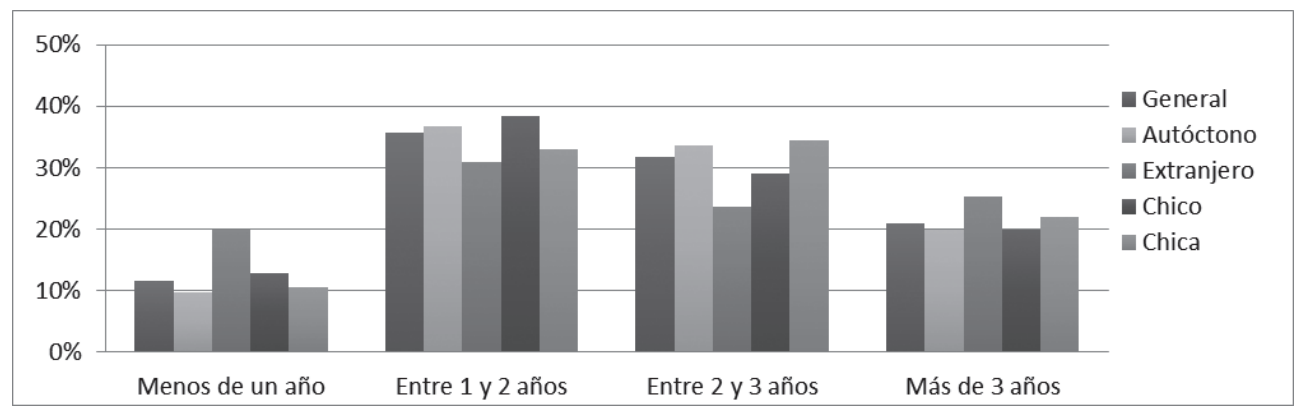

Con relación al tiempo semanal dedicado a las redes sociales, de lunes a viernes, cerca de la mitad de los alumnos (41.4\%) dedican menos de 1 hora a este medio, mientras que el $35.4 \%$ dedica de 1 a 3 horas, el $13.8 \%$ dedica más de 3 horas y solamente el 9.3\% afirma no dedicar tiempo a las redes sociales durante los días laborables. Los sábados y domingos el tiempo dedicado se incrementa y pasa a concentrar la mayoría de las respuestas entre las opciones "de 1 a 3 horas" (38.9\%) y "más de 3 horas" (23.7\%), disminuyendo el porcentaje de la opción "menos de 1 hora" (27\%) respecto al obtenido durante la semana, y manteniéndose similar la opción "ninguna hora" con un 10.4\%. Cabe resaltar que las horas dedicadas a las redes sociales durante el fin de semana es significativamente mayor en el caso de las chicas $\left(\chi^{2}(3)=31.325 \mathrm{p}=.000\right)$. Los resultados obtenidos confirman que más de una cuarta parte de las chicas $(27.7 \%)$ dedican más de tres horas frente a un $19.7 \%$ de los chicos.

En función del origen observamos diferencias significativas en el tiempo de acceso durante los fines de semana, a favor del alumnado autóctono, siendo el $24.4 \%$ de ellos y el $20.7 \%$ de extranjeros los que dedican más de tres horas a las redes, y el $40.3 \%$ de autóctonos y el $32.6 \%$ de extranjeros los que invierten entre 1 y 3 horas; por el contrario el 26.3\% de autóctonos y el $32.6 \%$ de extranjeros dedican menos de una hora y el $9 \%$ de autóctonos y $16.9 \%$ de extranjeros no dedican ninguna hora $(\chi 2(3)=33.804 ; \mathrm{p}=.000)$. 
En cuanto a las diferencias de uso de redes sociales entre alumnado con y $\sin \operatorname{NEAE}\left(\chi^{2}(3)=17.823 ; \mathrm{p}=.000\right)$, los resultados nos indican la existencia de un uso mayor por parte de los alumnos sin estas necesidades $(15.4 \%)$ con respecto a los que las tienen (9.3\%). Asimismo, la utilización durante más de tres horas es la opción seleccionada por el $24.5 \%$ de los alumnos sin NEAE y por el 20\% de los alumnos con NEAE. En consecuencia, comprobamos diferencias significativas si tenemos en cuenta las necesidades específicas de apoyo educativo, pues aquellos alumnos sin estas necesidades afirman, en un mayor número de ocasiones, que sí o probablemente sí beneficia el uso de las redes sociales $(57.2 \%)$ frente al $(47.4 \%)$ de los alumnos con NEAE $\left(\chi^{2}(3)=18.913 ; \mathrm{p}=.000\right)$ que no lo considera así.

\section{Relaciones de amistad}

Otra de las cuestiones planteadas a los alumnos hace referencia al número de amigos que los jóvenes tienen en las redes sociales. La gran mayoría de los encuestados $(60.8 \%)$ poseen más de 200 amigos, mientras que un $19.8 \%$ tiene entre 100 y 200 amigos y un $16.3 \%$ mantienen menos de 100 . Tan solo el 3.1\% declara no tener amigos en las redes sociales. En cuanto a la tipología de amigos con los que contactan suelen ser: sus amigos más cercanos (55.7\%), aquellos que hace tiempo que no ven (19.5\%) y los amigos de clase (14.7\%). Tan solo un $10.1 \%$ afirma utilizarlas para conocer gente nueva.

En referencia a las diferencias por género se comprueba que las chicas muestran mayor preferencia que los chicos (23.7\% frente al $15.4 \%)$ por contactar con gente que no ven hace tiempo. Además, si se trata de valorar el contacto con compañeros de clase comprobamos que hay diferencias significativas $\left(\chi^{2}(3)=49.575 ; \mathrm{p}=.000\right)$. Los resultados arrojan que existen porcentajes superiores en los chicos (18.5\%) respecto de las chicas (11\%). Si nos centramos en la nacionalidad, los datos reflejan que el porcentaje de alumnado autóctono es mayor cuando se trata de contactar con amigos cercanos. Sin embargo; estos porcentajes se invierten a favor del alumnado extranjero cuando hacemos referencia a contactar con gente nueva, gente que no ven hace tiempo o compañeros de clase $\left(\chi^{2}(3)=84.491\right.$; $\mathrm{p}=.000)$. Esta última situación se refleja por igual si tenemos en cuenta las necesidades educativas del alumnado, en cuanto que existe un mayor porcentaje de alumnos con NEAE (15.8\% frente al $8.9 \%)$ que prefieren usar las redes sociales para contactar con gente nueva $\left(\chi^{2}(3)=22.245 ; \mathrm{p}=.000\right)$. Si analizamos el numero de contactos en la red social, también se obtienen diferencias significativas $\left(\chi^{2}(2)=65.920 ; \mathrm{p}=.000\right)$. Los alumnos extranjeros tienen más de 200 contactos, aunque el porcentaje es menor que el obtenido por el alumnado autóctono. A pesar de ello, conviene resaltar que el 
porcentaje correspondiente a tener "ningún contacto" supone el 2.5\% para los autóctonos y más del doble para los extranjeros (5.6\%).

\section{Percepción sobre la dedicación}

En cuanto a la percepción que tiene el alumnado de quitarle tiempo a otras actividades para emplearlo en el uso de las redes sociales, un 68\% manifiesta que no quitan tiempo a nada. En el caso de aquellos que sí lo restan a otras actividades, prefieren hacerlo a los estudios (15.4\%), la familia (9.2\%) y amigos (7.3\%). Centrando nuestra atención en el hecho de desatender las tareas de estudio, por el tiempo invertido en utilizar las redes sociales, más de una tercera parte del alumnado comparte la idea de que no desatiende sus deberes nunca $(39.1 \%)$ y casi la misma proporción, pero aumentada, indica que casi nunca $(40.7 \%)$, lo que nos muestra la percepción positiva del alumnado a su organización del tiempo sobre el uso de las redes. Por otro lado, un $15.1 \%$ afirma desatenderlos casi siempre, y un $5.1 \%$ dice hacerlo siempre. Sin embargo, como refleja la figura 3 , existen diferencias significativas entre grupos en relación con esta cuestión $\left(\chi^{2}(3)=26.053 ; \mathrm{p}=.000\right)$. Los chicos quitan mayor tiempo a los estudios y a los amigos, mientras ellas consideran que desatienden más a las familias. Por otra parte, los autóctonos prefieren quitar en mayor porcentaje de tiempo a los estudios, mientras que los extranjeros prefieren quitárselo a los amigos $\left(\chi^{2}(3)=47.306 ; p=.000\right)$. En cuanto a las diferencias por grupos observamos un mayor porcentaje de utilización de las redes sociales por parte de los alumnos extranjeros $(10.4 \%)$ para la realización de trabajos $\left(\chi^{2}(3)=24.787\right.$; $\mathrm{p}=.000)$.

Si tenemos en cuenta las necesidades educativas, los alumnos con ellas prefieren restarle tiempo, en un mayor porcentaje que los alumnos sin NEAE, a los estudios y a los amigos $\left(\chi^{2}(3)=2.385 ; \mathrm{p}=.000\right)$.

Figura 3

El uso de las redes sociales resta tiempo

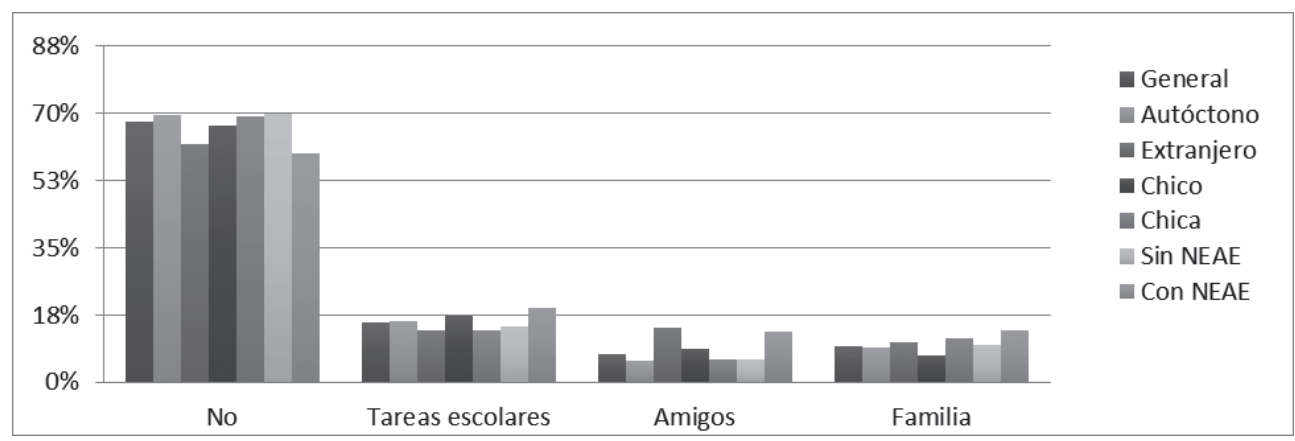




\section{Beneficios de las redes sociales}

Las redes sociales ejercen una gran atracción lúdica para los jóvenes por la facilidad con que les permite establecer relaciones sociales. En este sentido, más de la mitad de los participantes (53.1\%) afirman utilizar las redes sociales para divertirse y un $35.6 \%$ porque sus amigos están conectados. Asimismo, hay un pequeño porcentaje que dice utilizarlas para realizar trabajos de clase $(5.8 \%)$ o para compartir fotos principalmente $(5.5 \%)$.

Para complementar esta información quisimos indagar en esta cuestión, a través de la técnica Philips 66, y por ello formulamos dos preguntas a los distintos grupos seleccionados: ¿Por qué te divierten las redes sociales? y ¿Qué te ofrecen? Más de las tres cuartas partes de los alumnos participantes han respondido afirmativamente que las redes sociales les divierten y, solo un $12 \%$ contestan que no les divierte por el uso incorrecto que ellos puedan hacer de las mismas, o porque es una causa de distracción de sus tareas escolares. De todas las respuestas sobre lo que ofrecen las redes sociales, la de mayor frecuencia es la referida a las posibilidades de comunicación; entendida de diferentes modos, siendo la comunicación con amigos el uso más frecuente de las redes, aunque de forma excepcional pudiera servir para comunicarse con la pareja y con la familia. Además, les posibilitan los medios necesarios para compartir fotos, videos, o cualquier tipo de archivo, al igual que les permite conocer gente, buscar y visionar todo tipo de contenido audiovisual, convirtiéndose en fuente de entretenimiento e información, ya que les ofrecen la posibilidad de encontrar todos los juegos demandados actualmente por los jóvenes y poder jugar en línea con sus amigos o gente nueva a la que empiezan a conocer. Recogemos algunas respuestas de interés que demuestran tales afirmaciones:

"Porque conocemos nuevas cosas, subir fotos con frases, comentar opiniones sobre la gente, y conocer gente nueva. Porque nos aburrimos, permiten comunicarte con amigos y gente de lejos" (3- A15. C10)

"Porque nos ofrece información, me gustan porque algunas veces salen cosas muy divertidas y otras muy buenas. Además, están de moda y podemos comunicarnos con nuestros amigos y familiares" (4 ${ }^{\circ}$ - A7. C5).

"Información e interés porque y además es la moda de la sociedad $y$ todo el mundo quiere tener una solicitud en ellos" ( $3^{\circ}$ - A11. C4).

"Porque podemos hablar con amigos, se puede jugar a juegos, hacer videoconferencias, mandar fotos, vídeos, ubicaciones..." (3 - A 19. C6). 


\section{Consecuencias de la participación}

Planteando la posibilidad de que los alumnos permanecieran dos semanas sin redes sociales, el $70.1 \%$ considera que no pasaría nada e incluso un $4.7 \%$ reconoce que su vida cambiaría a mejor. Por el contrario, hay alumnos que no sabrían qué hacer en este caso (17.5\%), o creen que su vida cambiaría a peor $(7.7 \%)$. Ante el supuesto de estar dos semanas sin acceder a las redes sociales, los datos nos indican una mayor dependencia por parte de las chicas que de los chicos, en cuanto a las redes sociales, ya que el $11.1 \%$ de chicos y $23.3 \%$ de chicas no sabrían qué hacer ante la ausencia de las mismas $\left(\chi^{2}(3)=68.226 ; \mathrm{p}=.000\right)$.

Respecto a la relación con la familia, los amigos y las redes sociales, el $86.2 \%$ del alumnado afirma no haber tenido "nunca" o "casi nunca" discusiones con su familia por el uso de las redes sociales, pero encontramos que el 9.9\% afirman que "casi siempre" las tiene, y el 3.9\% contesta que siempre discute con su familia por este motivo. Son las chicas quienes tienen más discusiones con las familias por el uso de las redes sociales $\left(\chi^{2}(3)=72.303 ; \mathrm{p}=.000\right)$, tal y como se observa en la figura 4. Con los amigos, por el contrario, las discusiones son prácticamente inexistentes (el 87.6\% nunca ha discutido), aunque se comprueba que un 6.8\% sí lo hizo por subir fotos sin su permiso, por etiquetar $(2.7 \%)$, o por enviar mensajes ofensivos (2.9\%). En cuanto a las diferencias de género observamos que los porcentajes son similares, a pesar de que en esta ocasión los chicos son quienes discuten más y quienes, en mayor porcentaje han tenido discusiones por enviar mensajes ofensivos.

Figura 4

Discusiones por la utilización de las redes sociales

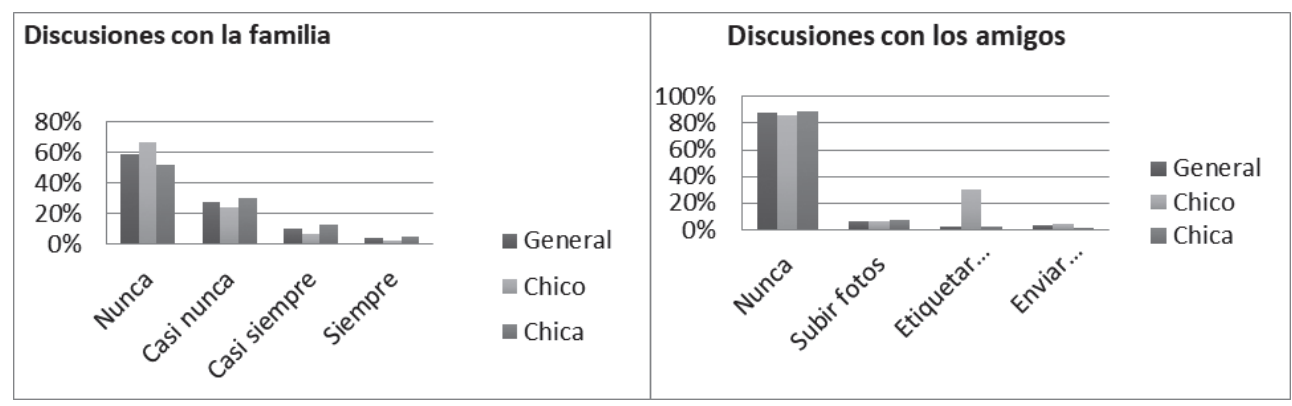

Por último, atendiendo a valorar las relaciones de nuestros estudiantes con los demás, y si creen que sus relaciones con los amigos se han visto mejoradas gracias a la participación en las redes sociales, se obtiene una respuesta dividida. Por un lado, aquellos que, rotundamente, dicen que sí se 
han visto mejoradas (14.4\%), quienes opinan que probablemente sí hayan mejorado (40.9\%) y los que dicen que no se han visto mejoradas $(26 \%)$, o probablemente no ha mejorado nada $(18.7 \%)$. Si analizamos los resultados en función de las necesidades educativas del alumnado comprobamos que las relaciones con los amigos debido al uso de las redes sociales, es menor en el caso de los alumnos con NEAE, pues el 33.1\% indica que este medio no le ha ayudado para mejorar sus relaciones con sus amigos cercanos, frente al $25.4 \%$ de los alumnos sin NEAE.

\section{DISCUSIÓN Y CONCLUSIONES}

De los resultados de nuestro estudio sobre la participación de adolescentes en las redes sociales se constata que el acceso, por parte del alumnado de $3^{\circ}$ y $4^{\circ}$ de ESO en los centros educativos de la Región de Murcia, está generalizado ya que solo un 10\% afirma no estar en ellas. Estos adolescentes son ya nativos digitales lo que hace que se inicien mucho antes en la construcción de su primer perfil social (Plaza de la Hoz, 2018).

El inicio temprano en las redes sociales es una realidad confirmada por los participantes de nuestro estudio, al reconocer que ya vienen utilizándolas desde hace algunos años y que su participación deja patente que, en general, las motivaciones no están orientadas únicamente a la esfera personal, también a cubrir las necesidades sociales de tipo afectivo y relacional (Bernal y Angulo, 2013; Colás et al., 2013; Notley, 2009) motivadas principalmente por intereses prosociales, constatándose diferencias individuales y de género (Asterhan \& Bouton, 2017).

En definitiva, a los jóvenes les atraen las redes sociales porque son un medio importante de comunicación sobre todo con sus amigos cercanos (muy pocos las usan para comunicarse con la familia o la pareja) y al mismo tiempo les posibilitan conocer gente nueva, destacando las posibilidades que ofrecen para compartir fotos y vídeos mediante este intercambio de imágenes que contribuye a la transformación en la manera de comunicarse (Svensson \& Russmann, 2017). Una realidad que refleja los deseos de una generación interactiva que tiene como aliadas afectivas a las redes sociales en su vida cotidiana, donde se entremezclan valores y sentimientos asociados a una estrecha unión entre ocio y entretenimiento, donde las gratificaciones experimentadas con el uso retroalimentan la motivación a seguir interaccionando (Dhir et al., 2018).

Con relación al primer objetivo específico referido a valorar la participación en el acceso y uso de los jóvenes atendiendo a la pertenencia a los grupos establecidos en nuestro estudio señalamos que siendo cierto que 
este inicio temprano en las redes sociales es una realidad generalizada, se constatan diferencias de género a favor de las chicas, y con relación al origen, se confirma una mayor participación de los autóctonos sobre extranjeros, al igual que es menor en aquellos que manifiestan tener necesidades específicas de apoyo educativo (NEAE).

En el caso del alumnado de origen extranjero, su participación es menor, aunque en cuanto al tiempo que llevan participando hay diferencias significativas respecto de los autóctonos, pues es mayor el porcentaje de alumnado extranjero que lleva más de 3 años, lo que puede deberse a la necesidad de mantener aproximación con su país de origen y utilizar la red social para conocer gente nueva.

En cuanto al acceso, no se aprecian diferencias significativas en función del género, aunque sí en cuanto al uso. Las chicas llevan más tiempo que los chicos en las redes sociales y les dedican más horas durante los fines de semana, por lo que son ellas quienes perciben un mayor temor, ante la posibilidad de quedarse sin este medio, al igual que manifiestan haber tenido, en más ocasiones, discusiones con amigos y familia por el tiempo empleado en ellas.

Del mismo modo, se constata que las chicas tienen una mayor preferencia que los chicos por contactar con gente que no ven desde hace tiempo, a diferencia de ellos que lo hacen más para contactar con los compañeros de clase. En este sentido, nuestros resultados confirman los de Fernández et al. (2015) al afirmar que las chicas usan más Internet para desarrollar las relaciones sociales. Las adolescentes tienden a ser más sociales que los chicos; aún así, el tener pocas relaciones recíprocas en las redes no afecta de manera negativa al desarrollo y bienestar adolescente; de igual manera que el mantener un amplio número de amigos en las redes no significa poseer altas capacidades comunicativas o de relación, ni tener un buen bienestar emocional (Li et al., 2017).

La participación en las redes sociales se mantiene de forma habitual; no hay que olvidar que la aparición del smartphone hizo incrementar el número de jóvenes con acceso a las redes (Viñals, 2016), aunque hay diferencias importantes entre aquellos que le dedican menos de una hora al día y otros que lo hacen entre 1 y 3 horas. Durante los fines de semana se incrementa el tiempo significativamente, encontrándose diferencias a favor de las chicas, y entre aquellos alumnos sin NEAE, en contra de aquellos de origen extranjero que invierten un tiempo menor que los autóctonos.

En cuanto al segundo objetivo específico Analizar las ventajas $e$ inconvenientes de la participación en las redes sociales, la participación 
en las redes sociales está asociada al entretenimiento y a la diversión, reconociendo que están empleando parte de su tiempo a una actividad que les satisface plenamente, con la facilidad que les proporciona una pantalla para socializarse e interactuar con los demás en cualquier momento (Bayer et al., 2016; Punyanunt-Carter et al., 2017), mientras que los jóvenes no perciben que, este modo de relacionarse y la dedicación del tiempo invertido a tal actividad, sea incompatible con la dedicada a otras tareas como son el estudio, la amistad o estar con la familia, por lo que se confirma que los jóvenes realizan un uso de las TIC, más asociado a un propósito comunicativo personal, enfocado a la diversión y al entretenimiento durante su tiempo de ocio, que a una práctica conducente al desarrollo formativo (Almansa et al., 2013; Colás et al., 2018).

En este sentido, la facilidad que proporcionan las redes sociales para establecer relaciones sociales es otro de los aspectos señalados para seguir participando en ellas. Más de la mitad de los estudiantes las utilizan para divertirse y por conectarse con sus amigos quedando muy por debajo la necesidad de búsqueda de información para desarrollar una actividad formativa. Sin embargo, llama la atención que su uso no ha sido un factor fundamental de mejora de las relaciones con sus amigos para todos los participantes, al comprobar que solo la mitad reconoce que sí han mejorado estas relaciones, existiendo diferencias con respecto a la percepción que tienen sobre la mejora de estas con amigos debido al uso de las redes sociales, siendo más negativa en el alumnado con NEAE, donde apreciamos que su campo de relaciones es menor.

Con respecto a la influencia negativa que pueda tener la participación en redes sociales, a la hora de mermar tiempo a otras actividades en los ámbitos del estudio, ocio y relación personal, no existe en general una percepción negativa que impida organizarse bien por lo que consideran que casi nunca quita tiempo a la realización de las tareas escolares. No obstante, conviene señalar que existe una cuarta parte de la muestra que reconoce la reducción al tiempo de estudio (chicos y autóctonos), a la relación con la familia (chicas) y a los amigos (autóctonos y alumnado con NEAE) admitiendo que pueden influir negativamente (Grion \& Manca, 2016), a la vez que son conscientes de que las redes sociales pueden restar tiempo para realizar las tareas escolares, siendo muy pocos quienes hacen uso de ellas para tal fin (García et al., 2013 ), siendo esta una implicación socieducativa de gran interés a tener en cuenta.

Atendiendo a las relaciones con la familia no existe una percepción preocupante entre el alumnado, aunque en ocasiones para un pequeño grupo pueda ser un motivo de discusión, en el caso de las chicas constatándose como discrepancias con los padres sobre el tiempo que dedican a las redes sociales. Sin embargo, se percibe una actitud mucho más permisiva y 
desigual que evidencia que la familia no es consciente de posibles riesgos (Livingstone, 2008; Livingstone \& Haddon, 2011). He aquí una propuesta de mejora, resultante de esta investigación, de cara a la formación de las familias en los centros educativos.

Como recomendación final consideramos que, ante la participación del alumnado en las redes sociales la educación no debe mirar para otro lado y asumir una labor orientadora para garantizar un buen uso de las tecnologías (Colás et al., 2018; Plaza de la Hoz, 2018; Vázquez y Cabero, 2015; Koh et al., 2019) reconociendo que debe realizar una actividad enfocada a la prevención educativa para el consumo de medios digitales ante una gran diversidad de alumnos y alumnas, con distintas necesidades educativas, procedentes de diversas culturas y situaciones socio-económicas, donde se tiene que garantizar la igualdad de oportunidades y su papel compensador, de las desigualdades (Livingstone et al., 2019; Martínez-Piñeiro et al., 2018).

Por último, queremos señalar algunas limitaciones de este artículo reconociendo, por un lado, que lo que presentamos es solo una parte de una investigación más amplia, tal y como se ha comentado en el apartado de Método; este hecho hace que existan cuestiones esenciales tratadas en otros trabajos previos y que no han sido abordadas en este momento. De igual forma, en relación con los resultados consideramos que no pueden generalizarse, como tal, debido a que la muestra ha recogido solo datos de la Región de Murcia y no de todos los estudiantes de Educación Secundaria sino de $3^{\circ}$ y $4^{\circ}$ de la ESO.

Por otro lado, a la hora de seleccionar al alumnado para la técnica cualitativa, se tuvieron que reducir a ese número, eligiendo cinco centros. Consideramos que, para poder obtener resultados concluyentes sobre este objeto de estudio, sería preciso ampliar el número de participantes en la Región de Murcia e incluso desarrollarlo en otras Comunidades Autónomas.

Para finalizar, consideramos que la investigación futura debería explorar más el fenómeno que hemos analizado, con observaciones directas y con otros instrumentos, para obtener una imagen más profunda y validar los hallazgos actuales, por lo que se necesitarán estudios cualitativos más detallados sobre las prácticas sociodigitales de nuestros adolescentes.

\section{NOTAS}

3 Las codificaciones empleadas han sido:

Nivel: $3^{\circ}-4^{\circ}$.

Alumno: $\mathrm{A} \mathrm{y} \mathrm{n}^{\circ}$ de la lista.

Centro: $\mathrm{C}$ y n ${ }^{\circ}$ del mismo. 
FRANCISCO JAVIER BALLESTA PAGÁN, JOSEFINA LOZANO MARTÍNEZ, MARI CARMEN CEREZO

\section{APOYOS}

Proyecto 15252/PPC/10. Convocatoria: Ayudas a la consolidación de grupos de investigación. Fundación Séneca. Agencia de Ciencia y Tecnología. Región de Murcia. 


\section{REFERENCIAS BIBLIOGRÁFICAS}

AIMC (2019). Navegantes en la Red: 21 Encuesta AIMC a usuarios de Internet. https://bit.ly/2CSiMXe

Almansa, A., Fonseca, O., y Castillo, A. (2013). Redes sociales y jóvenes. Uso de Facebook en la juventud colombiana y española. Comunicar, 20(40), 127-135. https://doi.org/10.3916/C40-2013-0303.

Asterhan, C.S., \& Bouton, E. (2017). Teenage peer-to-peer knowledge sharing through social network sites in secondary schools. Computers \& Education, 110, 16-3. https://doi. org/10.1016/j.compedu.2017.03.007

Bernal, C. y Angulo, F. (2013). Interacciones de los jóvenes andaluces en las redes sociales. Comunicar, 20(40), 25-30. https://doi.org/10.3916/ C40-2013-02-02

Bayer, J., Ellison, N., Schoenebeck, S., \& Falk, E. (2016). Sharing the small moments: ephemeral social interaction on Snapchat. Information Communication and Society, 19(7), 956-977. https://doi.org/10.1080/1369 118X.2015.1084349.

Boyd, D. \& Ellison, N. (2008). Social network sites: Definition, history, and scholarship. Journal of ComputerMediated Communication, 13, 210230. https://doi.org/10.1111/j.10836101.2007.00393.x

Colás, P., De Pablos, J., y Ballesta, J. (2018). Incidencia de las TIC en la enseñanza en el sistema educativo español: una revisión de la investigación. Revista de Educación a Distancia, 56. https://doi.org/10.6018/ $\mathrm{red} / 56 / 2$

Colás, P., González, T., y De Pablos, J. (2013). Juventud y redes sociales: motivaciones y usos preferentes. Comunicar, 20(40), 15-23. https://doi. org/10.3916/C40-2013-02-01

Dhir, A., Kaur, P., \& Rajala, R. (2018). Why do young people tag photos on social networking sites? Explaining user intentions. International Journal of Information Management, 38, 117-127. https://doi.org/10.1016/j. ijinfomgt.2017.07.004

Fernández, J., Peñalba, A., y Irazábal, I. (2015). Hábitos de uso y conductas de riesgo en Internet en la preadolescencia. Comunicar, 22(44), 113-120. https://doi.org/10.3916/C442015-12

Fundación Telefónica. (2019). Sociedad digital en España 2018. Fundación Telefónica. https://bit.ly/32Gh5Hh

García, A., López de Ayala, M.C., y Catalina, B. (2013). Hábitos de uso en Internet y en las redes sociales de los adolescentes españoles. Comunicar, 41, 195-204. https://doi.org/10.3916/ C41-2013-19

García-Ruiz, R., Ruiz, R.G., y Gómez, Á.H. (2018). Redes sociales y estudiantes: motivos de uso y gratificaciones. Evidencias para el aprendizaje. Aula Abierta, 47(3), 291-298. https://doi.org/10.17811/ rifie.47.3.2018.291-298

Garitano, E.T., Garrido, C.C., y Andonegui, A.R. (2019). Los hábitos de uso en las redes sociales de los preadolescentes. RIED. Revista Iberoamericana de Educación a Distancia, 22(2), 119-133. https://doi. org/10.5944/ried.22.2.23245

Garmendia, M., Jiménez, E., Casado, M.A., \& Mascheroni, G. (2016). Net Children Go Mobile: Riesgos y 
oportunidades en internet y uso de dispositivos móviles entre menores españoles (2010-2015). https://bit. ly/2OHmohz

Gewerc, A., Fraga, F., y Rodés, V. (2017). Niños y adolescentes frente a la Competencia Digital. Entre el teléfono móvil, youtubers y videojuegos. Revista Interuniversitaria de Formación del Profesorado, 31(2), 171-186.

Greenhow, C. \& Askari, E. (2017). Learning and Teaching with Social Network Sites: A Decade of Research in K-12 Related Education. Education and Information Technologies, 22(2), 623-645. https://doi.org/10.1007/ s10639-015-9446-9.

González Ramírez, T. y López Gracia, Á. (2018). La identidad digital de los adolescentes: usos y riesgos de las Tecnologías de la Información y la Comunicación. Revista Latinoamericana de Tecnología Educativa, 17(2), 73-85. https://doi. org/10.17398/1695-288X.17.2.73

Grion, V., \& Manca, S. (2016). Social network sites in secondary school: when students and teachers express their viewpoints. In ICERI2016 Proceedings (pp. 5992-6002).

Ibrahim, N.F., Wang, X., \& Bourne, H. (2017). Exploring the effect of user engagement in online brand communities: Evidence from twitter. Computers in Human Behavior, 72, 321-338. https://doi.org/10.1016/j. chb.2017.03.005

Instituto Nacional de Estadística, INE (2019). Población que usa Internet. https://goo.gl/OzpXOr

Koh, E., Jonathan, C., \& Tan, J.P.L. (2019). Exploring conditions for enhancing critical thinking in networked learning: Findings from a secondary school learning analytics environment. Education Sciences, 9(4), 287. https://doi.org/10.3390/ educsci9040287

Li, S., Hietajärvi, L., Palonen, T., Salmela-Aro, K., \& Hakkarainen, K. (2017). Adolescents' social networks: Exploring different patterns of sociodigital participation. Scandinavian Journal of Educational Research, 61(3), 255-274. https://doi.org/10.1080/00313 831.2015.1120236

Livingstone, S. (2008). Taking risky opportunities in youthful content creation: teenagers'use of social networking sites for intimacy, privacy and self-expression. New Media and Society, 10, 393-411. doi:10.1177/1461444808089415

Livingstone, S. \& Haddon, L. (2011). EU Kids Online III. A new project. LSE, EU Kids Online. https://bit.ly/3hkmeJd

Livingstone, S., Kardefelt-Winther, D., Kanchev, P., Cabello, P., Claro, M., Burton, P., \& Phyfer, J. (2019). Is there a ladder of children's online participation? https://bit.ly/2E7q227

Manca, S. \& Ranieri, M. (2017). Implications of Social Network Sites for Teaching and Learning: Where We Are and Where We Want to Go. Education and Information Technologies, 22(2), 605-622. https:// doi.org/10.1007/s10639-015-9429-x

Martínez-Piñeiro, E., Vila, E., y Gewerc, A. (2018). El papel de la familia en la construcción de la competencia digital. RISTI, 28, 1-13. https://doi. org/10.17013/risti.28.1-13

Notley, T. (2009). Young People, Online Networks, and Social Inclusion. Journal of Computer-Mediated Communication, 14(4), 1.2081.227. https://doi.org/10.1111/j.10836101.2009.01487.x 
Pacheco, B, Lozano, J., y González, N. (2018). Diagnóstico de utilización de Redes sociales: factor de riesgo para el adolescente. RIDE. Revista Iberoamericana para la Investigación y el Desarrollo Educativo, 8(16), 53-72. https://doi.org/10.23913/ride.v8i16.334

Plaza de la Hoz, J. (2018). Ventajas y desventajas del uso adolescente de las TIC: visión de los estudiantes. Revista Complutense de Educación. 29(2), 491-508. https://doi.org/10.5209/ RCED.53428

Punyanunt-Carter, N., De La Cruz, J., \& Wrench, J. (2017). Investigating the relationships among college students' satisfaction, addiction, needs, communication apprehension, motives, and uses and gratifications with Snapchat. Computers in Human Behaviour, 75, 870-875.

Rubio-Romero, J., Jiménez, J.M., y Barón-Dulce, G. (2019). Las redes sociales digitales como espacios de sociabilidad de los adolescentes. El caso del colegio Escolapios de Aluche. Revista Mediterránea, 10(2), 85-99. https://doi.org/10.14198/MEDCOM 2019.10.2.19

Sola, J.M., García, M., y Ortega, M. C. (2019). Las implicaciones del uso de dispositivos móviles en el proceso de enseñanza aprendizaje en alumnos de $5^{\circ}$ y $6^{\circ}$ de Primaria. Pixel-Bit, 55(7), 117-131. https://doi.org/10.12795/ pixelbit.2019.i55.07

Soler, I.R., López-Sánchez, C., y Lacave, T.T. (2018). Percepción de riesgo online en jóvenes y su efecto en el comportamiento digital. Comunicar, 56, 71-79. https://doi.org/10.3916/C562018-07

Svensson, J. \& Russmann, U. (2017). Introduction to visual communication in the age of social media: Conceptual, theoreti- cal and methodological challenges. Media and Communication, 5(4), 1-5. https://doi. org/10.17645/mac.v5i4.1263

Vázquez, A. y Cabero, J. (2015). Las redes sociales aplicadas a la formación. Revista Complutense de Educación, 26, 253-272. https://doi.org/10.5209/ rev_RCED.2015.v26.47078

Vizcaíno-Laorga, R., Catalina-García, B., \& de Ayala-López, M. (2019). Participación y compromiso de los jóvenes en el entorno digital. Usos de las redes sociales y percepción de sus consecuencias. Revista Latina de Comunicación Social, (74), 554-572. https://doi.org/10.4185/RLCS-20191345

Viñals, A (2016). El Ocio conectado, móvil, transmedia y multisoporte de los jóvenes en la Era Digital. Fonseca, Journal of Comunication, 13(13), 99-113. https://doi.org/10.14201/ fjc20161399113

Yau, J.C. \& Reich, S.M. (2018). It's just a lot of work: Adolescents' elfpresentation norms and practices on facebook and instagram. Journal of Research on Adolescence, 1-14. https:// doi.org/10.1111/jora.12376 


\section{PERFIL ACADÉMICO Y PROFESIONAL DE LOS AUTORES}

Francisco Javier Ballesta Pagán. ORCID: http://orcid.org/0000-00027830-5053

Profesor Titular de Universidad en el Departamento de Didáctica y Organización Escolar de la Universidad de Murcia. Desarrolla su actividad investigadora en Tecnología Educativa donde ha dirigido y colabora en la realización de diversos proyectos de investigación sobre comunicación educativa, consumo de medios digitales y formación en TIC. E-mail: pagan@ um.es 7896

Josefina Lozano Martínez. ORCID: http://orcid.org/0000-0003-4966-

Profesora Titular de Universidad en el Departamento de Didáctica y Organización Escolar de la Universidad de Murcia. Ha realizado trabajos de investigación sobre la educación inclusiva, intercultural y la atención a la diversidad en términos generales, y tiene publicaciones y comunicaciones sobre esta temática. Sus líneas de investigación actuales son la educación intercultural, el consumo de medios digitales y la escuela inclusiva. E-mail: lozanoma@um.es 6149-3863

Mari Carmen Cerezo Máiquez. ORCID: http://orcid.org/0000-0001-

Profesora Asociada del Departamento de Didáctica y Organización Escolar de la Universidad de Murcia. Maestra de Educación Primaria, Licenciada en Pedagogía y Psicopedagogía por la Universidad de Murcia. Desarrolla su actividad investigadora centrada en la educación intercultural, el consumo de medios digitales y la escuela inclusiva. E-mail: mcarmen. cerezo@um.es

Irina Sherezade Castillo Reche. ORCID: http://orcid.org/0000-00017965-1567

Profesora Asociada del Departamento de Didáctica y Organización Escolar de la Universidad de Murcia. Licenciada en Pedagogía por la Universidad de Murcia. Desarrolla su actividad investigadora centrada en la educación intercultural, la educación de alumnado con necesidad específica de apoyo educativo, el consumo de medios digitales y la escuela inclusiva. E-mail: irinasherezade.castillo@um.es 
Fecha Recepción del Artículo: 21. Febrero. 2020

Fecha Modificación del Artículo: 02. Julio. 2020

Fecha Aceptación del Artículo: 03. Julio. 2020

Fecha Revisión para Publicación: 08. Julio. 2020 